\title{
Influencia de los estereotipos de género asociados con la violencia contra las mujeres
}

Influence of gender stereotypes associated to violence against women

Óscar Antonio Sánchez-Velásquez ${ }^{3}$

Docente

oscar.sanchez@mail.utec.edu.sv

\section{Resumen}

Se trata de un ejercicio académico en el marco de la cátedra Realidad Nacional, en el cual se aborda la temáticaproblemática de la influencia de los estereotipos de género y su relación con los diferentes tipos de violencia contra las mujeres. Se determina cuáles son y en qué consisten los estereotipos de género. De igual manera se esclarecen las diferentes categorías de violencia contra las mujeres y la terminología fundamental para el entendimiento de la temática. Con esta base se parte para identificar las diferentes cosmovisiones y percepciones que tienen los estudiantes de una asignatura común, en este caso, Seminario Taller de Competencias de la Universidad Tecnológica de El Salvador respecto al objeto de estudio.

\section{Palabras clave}

Discriminación sexual contra la mujer; Derechos de la mujer; Violencia contra la mujer

\section{Introducción}

Los estereotipos de género más complejos pueden ejercer un efecto negativo en las conductas de hombres y mujeres, conduciéndolos a ciclos de violencia que, hasta cierto punto, para ellos son normales y aceptables en la sociedad.

\begin{abstract}
As an academic assignment in the National Reality course, this research deals with the influence of gender stereotypes and how they relate to the different types of violence against women. It describes which gender stereotypes exists and what they consist of. Likewise, it clarifies the different existing categories of violence against women and the basic terminology for the understanding of this topic. This lays the foundation for the identification of the several world views and perceptions that students in an elective course at Universidad Tecnológica de El Salvador have, as it is the case of the Competencies Seminar, in regards to the object of study.
\end{abstract}

\section{Keywords}

Sexual discrimination against women; Women's rights; violence against women

El uso de los estereotipos de género es la práctica de asignar a una persona determinad, atributos, características o funciones específicas únicamente por su pertenencia al grupo social masculino o femenino. La utilización de los estereotipos de género es dañina cuando genera violaciones de los derechos y las libertades fundamentales. 
El derecho internacional de los derechos humanos asigna a los Estados la obligación de eliminar la discriminación y la violación de los derechos en contra hombres y mujeres en todos los ámbitos de la vida. Esta obligación exige que los Estados adopten medidas para abordar los estereotipos de género, tanto en la esfera pública como en la privada, así como para evitar la utilización de dichos estereotipos.

Los Estados entonces deberán tomar todas las medidas apropiadas para modificar los patrones socioculturales de conducta de hombres y mujeres, con miras a alcanzar la eliminación de los prejuicios y las prácticas sexistas frecuentes y de cualquier otra índole que estén basadas en la idea de la inferioridad o superioridad de cualquiera de los sexos o en funciones estereotipadas de hombres y mujeres.

Este estudio tiene como finalidad identificar los estereotipos de género en los cuales se sustentan las conductas violentas en contra de las mujeres y la influencia que estos tienen en el desarrollo de sus vidas.

De acuerdo con los datos presentados por el Instituto de Medicina Legal (IML), la mayor incidencia de violencia física contra las mujeres se presenta en el ámbito familiar (6.761), habiendo una diferencia de 2.510 casos de reconocimiento de lesiones entre este ámbito y las ocurridas a consecuencia de hechos considerados de violencia común.

Este dato demuestra la gravedad de la situación de violencia que las mujeres enfrentan, ya que en la mayoría de casos se ven vulneradas en su integridad física en espacios que deberían ser considerados seguros y con acciones cometidas por personas con las que se mantiene una relación de confianza.

Según el informe del IML, tanto en los casos ocurridos por violencia común como por violencia intrafamiliar, hay un alto número de hechos que ocurren en casa de la víctima o casa conocida. Para el año 2014, según datos publicados por el IML en el Portal de Transparencia de la Corte Suprema de Justicia, de 2.943 reconocimientos practicados a mujeres víctimas de violencia física en el ámbito familiar, 2.008 delitos ocurrieron en casa propia y 211 en casa conocida.

Conforme los datos del IML, los departamentos en los que más casos de violencia física se han registrado para el período enero 2014-marzo 2015 son San Salvador (37,65\%), seguido por Santa Ana (10,95 \%) y Sonsonate (10,33 \%). Morazán es el departamento con menos hechos de violencia física reportados $(1,33 \%)^{2}$

La necesidad de garantizar el respeto a la mujer y evitar las conductas que conducen a la violencia a la que se ven sometidas las mujeres, siendo una grave vulneración a sus derechos, es una constante que ha llevado a los organismos internacionales y las instituciones gubernamentales a adoptar normas y medidas encaminadas a la erradicación de la violencia contra la mujer.

A través de la Declaración sobre la Eliminación de la Violencia contra la Mujer, de la Organización de las Naciones Unidas, se ha reconocido que "la violencia contra las mujeres constituye un obstáculo para el logro de la igualdad, el desarrollo y la paz"; además de ser "una violación de los derechos humanos y las libertades fundamentales, así como también constituye una manifestación de relaciones de poder históricamente desiguales entre el hombre y la mujer".

Por esta razón, el país cuenta con programas de atención integral para mujeres que enfrentan la violencia, a través del Instituto Salvadoreño de Desarrollo de la Mujer (Isdemu), por medio de las siguientes oficinas de atención: ${ }^{3}$

- Centro de llamadas 126

- Centro integral de atención Isdemu

- Unidades de atención especializada en las sedes de Ciudad Mujer

- Unidades de atención en centros judiciales

- Unidades de atención departamentales

- Ventanillas móviles y fijas

- Sistema de protección a mujeres víctimas de violencia

De acuerdo con datos consensuados por la Policía Nacional Civil, el IML y la Fiscalía General de la República, de enero 2012 a junio 2015, se han registrado 1.062 muertes violentas de mujeres. Entre enero y junio de 2015 se han cometido 230 casos de muertes violentas de mujeres, generando para este período una tasa de 6.73 por cada 100.000 habitantes. Esta es una tendencia al alza, considerando que en el primer semestre del 2015 la cantidad de muertes violentas de mujeres representa $78 \%$ de los casos registrados durante todo el 2014.

2 Isdemu. "Informe sobre el Estado y Situación de la Violencia contra las mujeres en El Salvador", p. 8.

3 Idem, p. 12 
De enero de 2012 a junio de 2015, en el rango de edad de 10 a 39 años, se han registrado 681 casos de muertes violentas de mujeres, que representan el $64 \%$ del total. Se mantiene una tendencia constante en el hecho de que son mayoritariamente las mujeres jóvenes las más expuestas a ser asesinadas, especialmente en el rango de 10 a 29 años.

Para ese mismo período, los tres tipos de arma más utilizadas fueron: de fuego (en el 60,2 \% de los casos) y corto contundente (en el $12,1 \%$ de los casos); y asfixia por estrangulación (en el 8,2 \% de los casos). El IML recoge dentro de la categoría Agresiones sexuales los reconocimientos realizados en hechos considerados como violación, otras agresiones sexuales y estupro. De los 9.290 reconocimientos practicados desde la entrada en vigencia de la Ley Especial Integral para una Vida Libre de Violencia para las Mujeres hasta marzo de 2015, las mayores concentraciones de los casos fueron cometidos contra niñas y adolescentes de entre o y 19 años, pudiéndose establecer las relaciones siguientes:

- A mayor edad, menos casos de agresión sexual, lo que constituye un indicador de cómo la edad puede constituirse en un factor que incrementa la vulnerabilidad de las mujeres a ser víctimas de violencia sexual.

- El embarazo en niñas y adolescentes constituye una violación al derecho a una vida libre de violencia y al ejercicio de los derechos sexuales y reproductivos reconocidos en el ordenamiento jurídico salvadoreño. ${ }^{4}$

\section{Método}

El método que se ha utilizado en este estudio es el cuantitativo, conocido como estadístico o hipotético deductivo, con el cual, mediante la frecuencia de datos obtenidos, puede inferirse 0 generalizarse la opinión de una muestra seleccionada con la población o muestra. El uso de este método es comprobar la teoría. En otras palabras, se trata de cotejar o establecer una relación entre constructos teóricos existentes (abstracción) y lo identificado en la parcela, segmento o porción de realidad sujeta de estudio (concreción).

El primer paso que se realizó fue una investigación documental, en la que se recopiló información relacionada con el tema de los estereotipos de género y la violencia contra la mujer para poder definir el problema de la investigación y orientarlo hacia los objetivos.
Posteriormente, se seleccionó la encuesta como la técnica que se utilizaría para la investigación y se diseñó el cuestionario como instrumento para la recolección de la información.

Tipo de estudio

El tipo de estudio realizado en la investigación fue de carácter descriptivo-transversal. Descriptivo porque se especificaron las características, opiniones, percepciones y cosmovisiones de los individuos seleccionados respecto al tema. En términos de temporalidad es de tipo transversal, ya que arranca en abril de 2017 y cierra en mayo del mismo año.

\section{Grupo de estudio}

El grupo de estudio fue de 80 alumnos de la sección 04 de la asignatura Seminario Taller de Competencias de la Universidad Tecnológica de El Salvador.

Las características de los informantes fueron las siguientes:

- Edades $\quad=18$ a 45 años

- Género $\quad=37$ mujeres y 43 hombres

- Zona = 71 urbana y 9 rural

- Estado civil = 70 solteros, 6 casados, 4 acompañados

- Nivel socioeconómico = 14 bajo, 63 medio y 3 alto

\section{Resultados}

Referente a la percepción de los estudiantes respecto a los estereotipos de género, se puede decir que, a lo largo del análisis de los resultados, se ha detectado una clara diferencia entre las respuestas de mujeres y hombres, lo que significa que la muestra ha respondido de manera estereotipada en general.

Los resultados obtenidos dan valores significativos que indican que tanto hombres como mujeres están todavía estereotipados por el género, demostrando por medio de sus opiniones la manera de como ellos consideran que deben ser las cosas, conservando las ideas estructuradas de la sociedad.

Los estereotipos de género interiorizados en los individuos sujetos de estudio limitan el desarrollo de la personalidad, tanto femenina como masculina, ya que sus compartimientos y actitudes, seguramente, obedecen al sexo al que pertenecen.

\footnotetext{
${ }^{4}$ Op cit, p. 10.
} 
Respuestas femenino

\section{Las mujeres son inferiores a los hombres.}

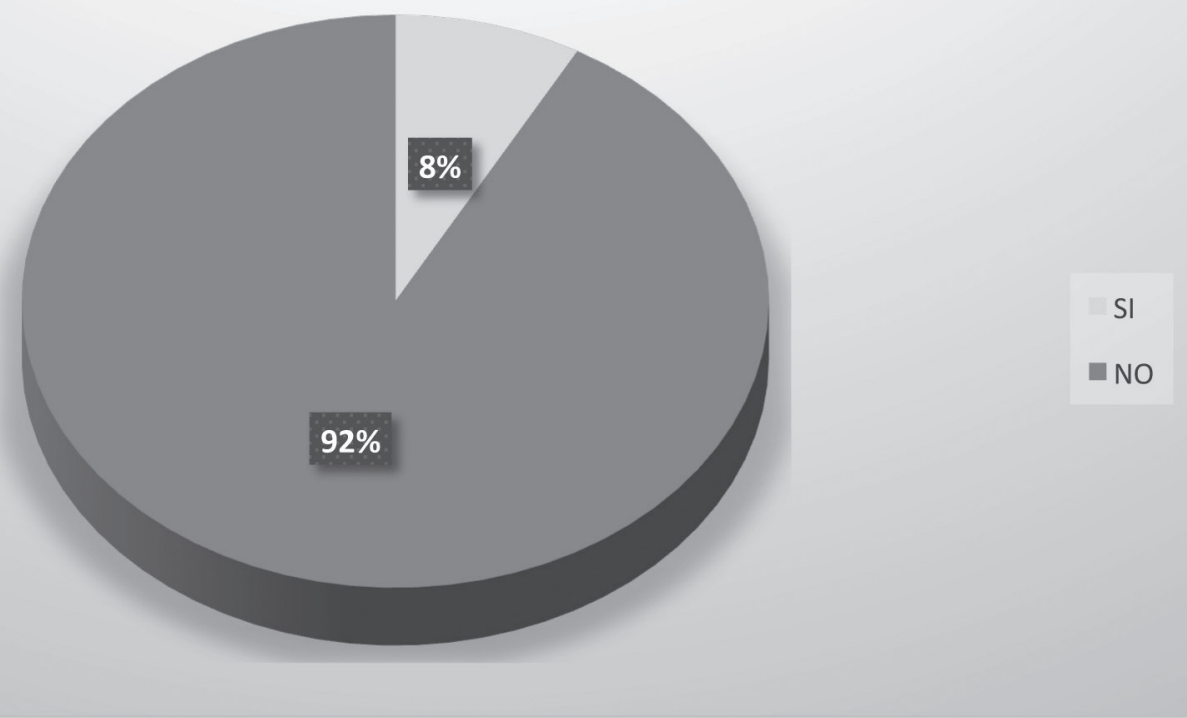

Respuestas masculino

\section{Las mujeres son inferiores a los hombres.}

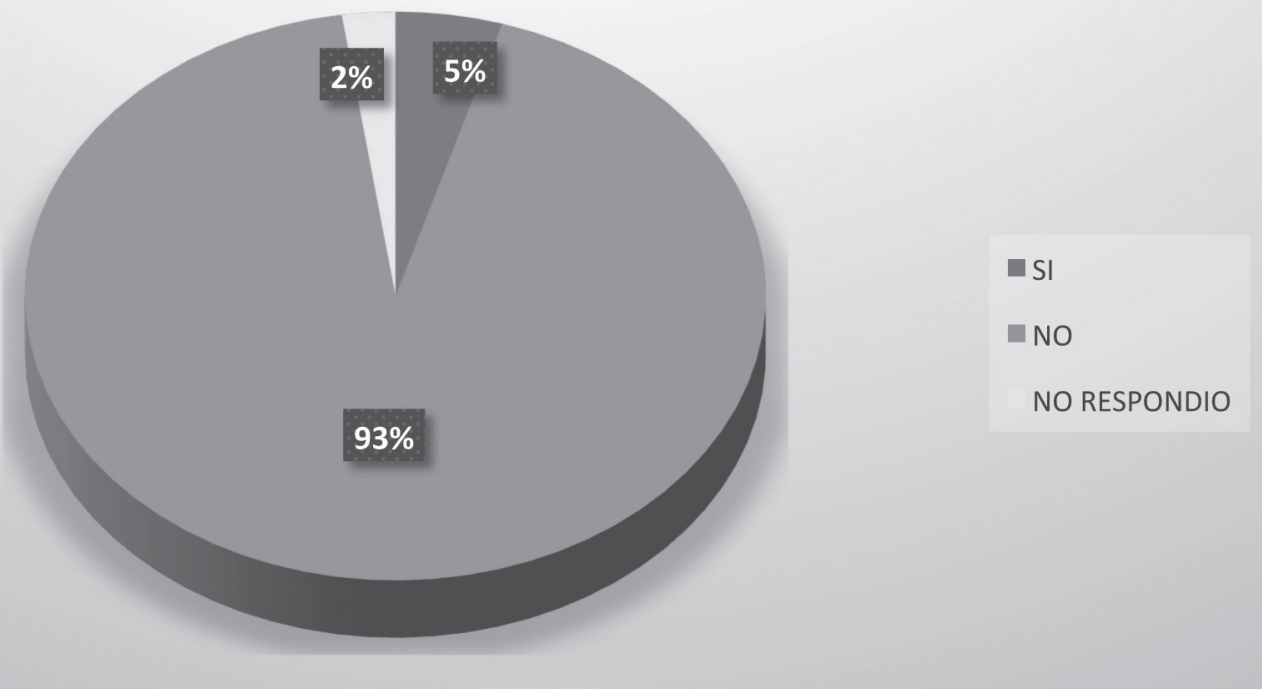

La pregunta 1 nos refleja que aún persiste en el pensamiento de hombres y mujeres un estereotipo de género de dominio patriarcal que promueve la desigualdad, desvalorizando a la mujer a un plano inferior. 
Respuestas femenino

\section{Si el marido es el que aporta el dinero en casa, la mujer debe estar sometida a él.}

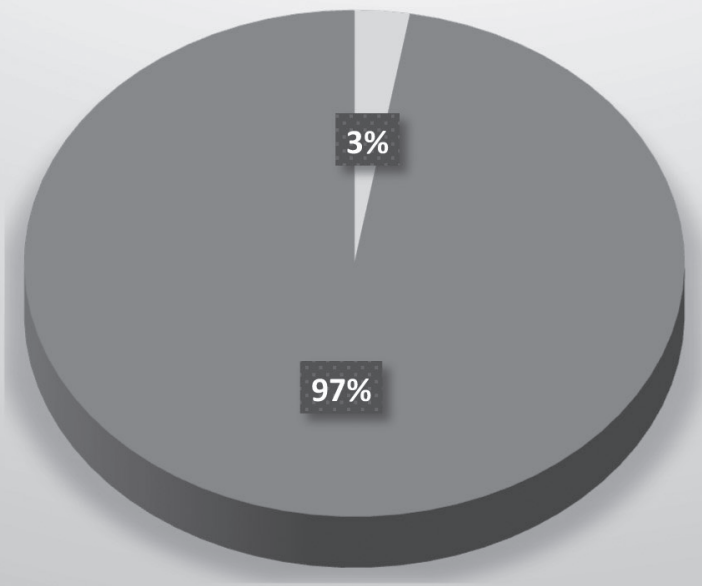

\section{Si el marido es el que aporta el dinero en casa, la mujer debe estar sometida a él.}

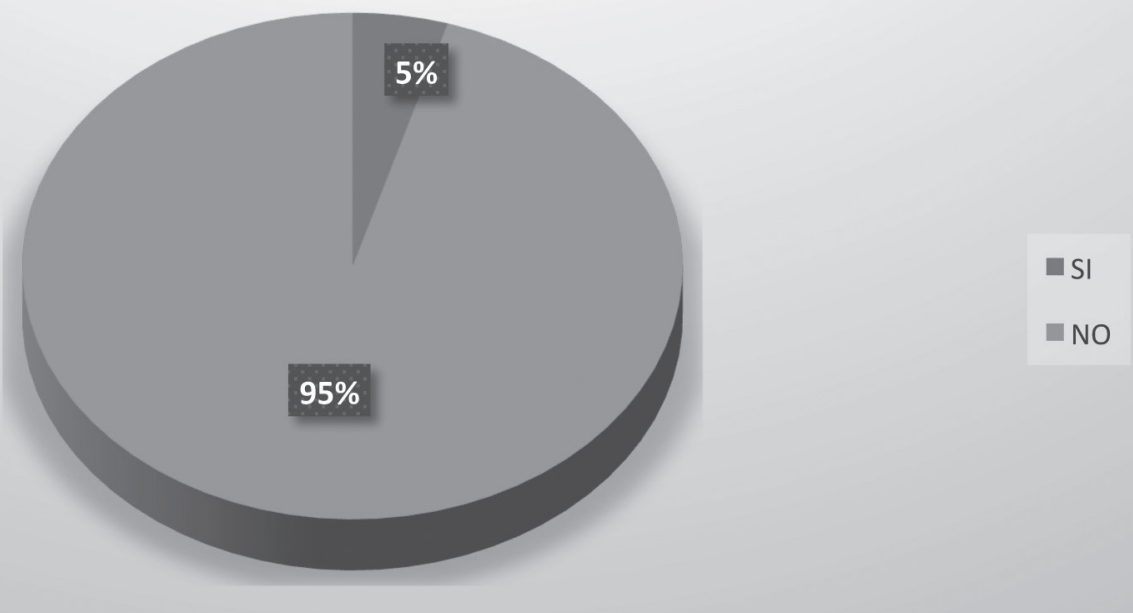

La pregunta 2 nos revela nuevamente que, aunque en un porcentaje pequeño, la idea del estereotipo del dominio del hombre sobre la mujer promueve el sometimiento por factores económicos, llevándonos al resultado de la desigualdad. 
Respuestas femenino

\section{La obligación de una mujer es tener relaciones sexuales con su marido, aunque ella no quiera.}

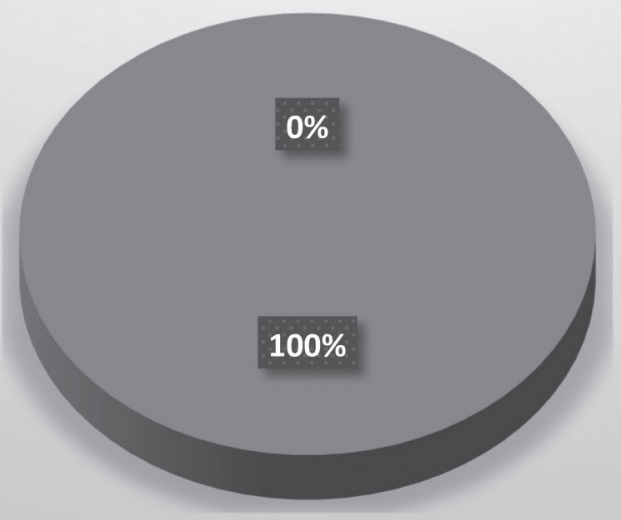

\section{La obligación de una mujer es tener relaciones sexuales con su marido, aunque ella no quiera.}

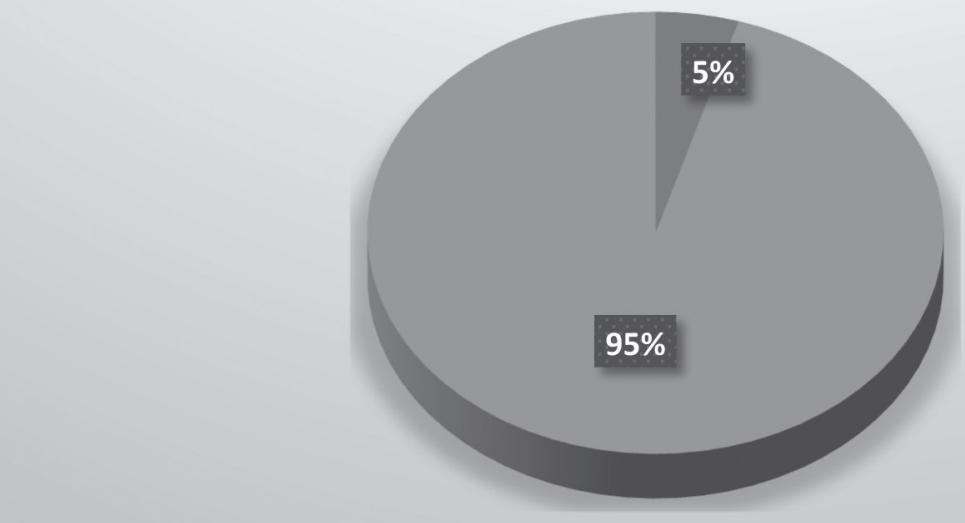

La pregunta 3 nos revela que la mujer ya ha entendido sus derechos de decidir voluntariamente su vida sexual, pero que en el caso de los hombres un porcentaje persiste en la idea estereotipada de dominio sobre la mujer en todo sentido, aunque le violente sus derechos. 


\section{Una mujer no debe llevar la contraria a su marido.}

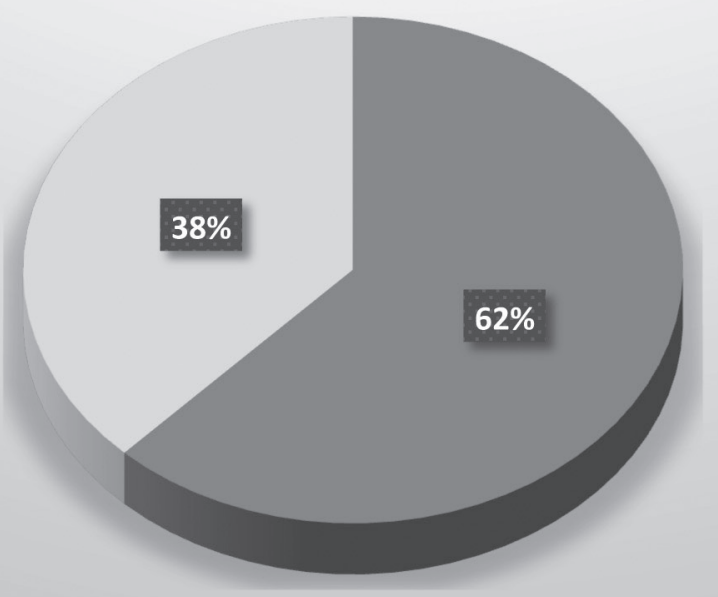

Respuestas masculino

\section{Una mujer no debe llevar la contraria a su marido.}

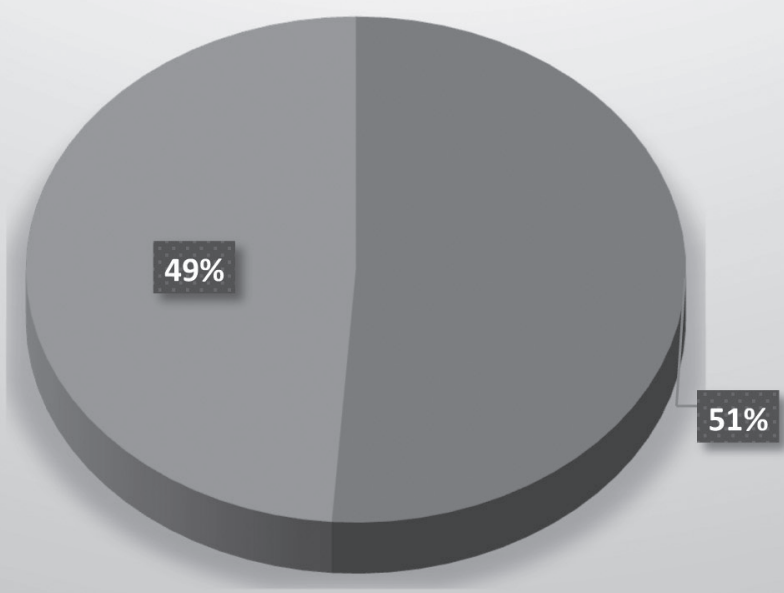

La pregunta 4 nos refleja el pensamiento de que tanto hombres como mujeres coinciden en que la mujer debe ser sumisa. 


\section{Análisis}

Los estereotipos asociados con la violencia hacia las mujeres que se identificaron con el instrumento de investigación fueron los siguientes:

- Dominio del hombre sobre la mujer.

- Desigualdad entre hombres y mujeres.

- El marido tiene derecho de ejercer el control sobre la mujer.

- El hombre es quien manda por dar el mantenimiento al hogar.

- La mujer es inferior al hombre.

- La mujer siempre debe ser sumisa.

- El maltrato de los hombres es muestra de amor hacia la mujer.

- Los hombres son violentos por naturaleza y la mujer debe callar.

- Los celos son muestra de afecto.

- La violencia es el medio para resolver los conflictos.

- La mujer debe depender del hombre.

- El hombre es quien debe tomar las decisiones.

- Lo que sucede dentro de la pareja es privado, nadie tiene derecho a entrometerse.

- La mujer maltratada tiene la culpa de que el hombre la maltrate.

- El hombre maltrata a la mujer porque algo le ha hecho ella.

\section{Discusión}

1. Entre la lógica binaria violencia-convivencia impactará esta última a partir de las diferentes intervenciones de prevención y control de los distintos tipos de violencia.

2. Si realmente las diferentes leyes y normativas de prevención y ataque a la violencia reducirán las diversas manifestaciones de violencia.

3. Puede inferirse la percepción de los estudiantes de la UTE respecto a los estereotipos de género con la población salvadoreña en general.

\section{Referencias}

Diccionario etimológico español en línea (2017). “Origen de las palabras". Obtenido de http://etimologias.dechile.net/

"Estereotipos de género". Recuperado de http://www. webconsultas.com/bebes-y-ninos/psicologia-infantil/ estereotipos-de-genero

Gestoso, J.I. (1993). Los estereotipos sociales. Madrid: Editorial Complutense.

Isdemu (2015). "Informe sobre el estado y situación de la violencia contra las mujeres en El Salvador". San Salvador: Aecid.

Naciones Unidas (2014). "Los derechos de la mujer son derechos humanos". New York: ONU.

Organización Mundial de la Salud (2002). "Política de la OMS en materia de género". En G. H. Brundtland.

Real Academia Española (2017). Diccionario de la lengua española. Obtenido de http://dle.rae.es/?id=GqSjqfE 